
\title{
25 Research Square \\ Complementary Feeding: Is It The Forgotten Factor of The First 1000 Days of Life?
}

Catherine Muthoni Njeru ( $\nabla$ njerucathy@gmail.com )

Gertrude's Children's Hospital, Muthaiga https://orcid.org/0000-0002-2417-3120

Anthony Ngugi

Aga Khan University Hospital

Caroline Kathomi

Aga Khan University Hospital

Mary Slessor Limbe

Aga Khan University Hospital

Research

Keywords: Complementary feeding, World Health Organisation, Guidelines, Nutrition, Overweight, Infant, Caregiver

Posted Date: January 8th, 2021

DOI: https://doi.org/10.21203/rs.3.rs-140037/v1

License: (1) (1) This work is licensed under a Creative Commons Attribution 4.0 International License. Read Full License 


\section{Abstract}

\section{Background:}

Appropriate complementary feeding is a practice with great potential for reduction of nutrition-related diseases and mortalities in children. However, uptake of the recommended World Health Organisation (WHO) infant and young child feeding practices in Kenya remains low. Most previous studies have been done in rural or urban poor populations hence the need to provide practical guidelines for the complementary feeding period in a typical middle-class urban setting.

This study investigated complementary feeding practices by caregivers of infants and young children on follow up at Aga Khan University Hospital-Nairobi (AKUH-N) well-baby clinic and the factors influencing compliance with the WHO guidelines.

\section{Methods:}

A total of 290 caregivers of infants and young children aged between nine and twenty-four months were interviewed using a questionnaire. Out of these, 21 were purposively sampled to participate in a qualitative study using in-depth interviews. Thematic analysis was used to analyse qualitative data while descriptive statistics, univariate and multivariate regression analysis were used for quantitative data. A convergent parallel design was used to integrate the qualitative and quantitative data.

\section{Results:}

Out of 290 caregivers interviewed, 125 (43.1\%) complied with WHO complementary feeding guidelines. Proportions of underweight and stunting were $6.9 \%$ and $1.7 \%$, respectively, while that of overweight was $8.6 \%$. Factors that affected complementary feeding practices included education level of the caregiver, access to information, support from healthcare workers and fear of allergic reactions.

\section{Conclusions:}

Full compliance to WHO complementary feeding guidelines remained below $50 \%$. In addition, the proportion of children who were overweight in this study was double the national average, highlighting a growing trend of obesity in childhood. Some of the factors positively associated with compliance with complementary feeding guidelines included access to multiple sources of information, and support from health workers and family members. Lack of tertiary education of the caregiver, absence of specific and practical guidelines, resource constraints and fear of allergies were found to negatively impact compliance with complementary feeding guidelines.

\section{Introduction}

Nutrition, is of great importance in the health and well-being of a child, especially in the period from conception to the age of two years. Malnutrition not only causes death in millions of children, but also 
leaves many more with lifelong physical and mental impairments (1). In-utero, the growing fetus is completely dependent on the mother for its nutrition. From birth to about six months of age, adequate intake of breast milk meets the nutritional requirements for full term infants. Thereafter, complementary feeds are needed in order to meet the nutritional and developmental needs of the growing infant. World Health Organization (WHO) defines appropriate complementary feeding as: timely, adequate, safe and properly fed.

Worldwide, low compliance with these recommendations is evident (2). It is concerning that complementary feeds are either introduced too early or too late and even then, they are often not of the appropriate quality or quantity. Adequacy of feeds is guided by the WHO definition of minimum acceptable diet, which requires that both minimum meal frequency and minimum diet diversity be met (3). Minimum meal frequency is defined as "two times per day for breastfed infants aged six to eight months, three times for breastfed children aged nine to twenty-four months and four times for nonbreastfed children aged six to twenty-four months." This indicator is intended as a surrogate for energy intake from foods other than breast milk (4).

Minimum Diet Diversity is a diet consisting of foods from four or more of the following food groups: grains, roots and tubers; legumes and nuts; dairy products (milk, yogurt, fermented milk); flesh foods (meat, fish, poultry and liver/organ meats); eggs; vitamin-A rich-fruits and vegetables; other fruits and vegetables (WHO Publication, 2008a).

The core indicators that need to be met for compliance are as follows: timely introduction of complementary feeds (at 6 months), adequacy of feeds (meeting both the minimum frequency and minimum diet diversity), inclusion of iron-rich foods in the diet and continued breastfeeding on demand until at least two years of age (3).

The latest Kenya Demographic and Health Survey (KDHS)statistics indicate that only $22 \%$ of children are fed in accordance with the WHO-recommended infant and young child feeding practices (5). Most studies in Kenya have focused on infants attending clinics at public facilities (Otaigo, M. L. et al., 2014, Bakalemwa R et al., 2014, Kimani-murage et al., 2011). They found that low socioeconomic status as well as lack of provision of nutrition education were barriers to proper complementary feeding practices.

Nutrition education, which has been shown to improve complementary feeding practice, is done regularly by the paediatricians and nurses at the Aga Khan University Hospital-Nairobi (AKUH-N)well-baby clinics. Despite this, trends towards growth-faltering and over-weight have been observed, pointing to both underand over-nutrition. A retrospective chart review of 48 infants seen at the well-baby clinic revealed that 21 $(44 \%)$ of the infants had weight-for-age growth-faltering while $3(6 \%)$ were overweight.

Whereas other studies have focused on rural or socio-economically disadvantaged populations, AKUH-N offers a unique opportunity for exploration of other factors and gaps for intervention relevant in an urban cosmopolitan middle-class population. 


\section{Methods}

\subsection{Study Area and Population}

The study was carried out at the AKUH-N well-baby clinic. This is a tertiary, private non-profit institution established in 1958. It has a 254-bedcapacity and provides quality inpatient medical services, general and specialist outpatient clinics supported by comprehensive diagnostic services. The well-baby clinic provides growth and development monitoring of children of up to two years. It also provides health education, including advice on proper complementary feeding methods, as well as vaccination services. The study targeted infants between nine and twenty-four months of age who were on follow-up at this clinic, and their primary caregivers.

\subsection{Study Design}

This was a mixed-methods cross-sectional study that combined both quantitative and qualitative methods in a convergent parallel design.

\subsection{Data Collection}

\subsubsection{Quantitative Component}

Quantitative sample size calculation was based on the national rate of compliance to WHO guidelines reported at $22 \%$ (5). Two hundred and ninety respondents were recruited for the quantitative arm of the study. A questionnaire was used to measure knowledge, attitude and practice of complementary feeding against the WHO set standards (8). It consisted of four sections with a total of 50 questions. The first three sections recorded information on the interview details, demographic characteristics of the caregiver and characteristics of the child respectively. The final section had questions on knowledge and attitude to complementary feeding recommendations. The questionnaire was largely adapted from the WHO Indicators for assessing Infant and Young Child Feeding Practices measurements booklet (3). The 20 questions in the section on knowledge of WHO guidelines on complementary feeding practices were adapted from recommendations given in the summary of guiding principles on complementary feeding (8).

Three interviewers were trained on how the tools were developed, how to approach respondents, how to administer the questionnaires and check data. The questionnaire was piloted among 10 respondents who were not included in the study to check for clarity, flow and ease of administration of the questions. No revisions were found necessary after the piloting process. Following written informed consent, they administered the questionnaire to the caregivers in an interview that took approximately 15 minutes. The principal investigator(CN) supervised this process to ensure that the questionnaires were being properly administered. Data were collected from March to December 2018.

All infants whose caregivers responded to the questionnaire had their growth parameters plotted in standard WHO weight-for-age, length-for-age and weight-for-height growth charts. Infants who were of 
appropriate weight, overweight, underweight/those with growth-faltering were then identified.

\subsubsection{Qualitative Component}

An interview guide which consisted of nine sections with a total of 15 questions was used to collect qualitative data. It assessed predisposing, enabling and reinforcing factors affecting compliance with WHO guidelines for complementary feeding. This was based on the PRECEDE model by Green and Kreuter( Green LW, Kreuter MW, 2005)-a community oriented participatory model for investigating factors affecting health promoting behaviour.

The first seven caregivers in each category whose infants were classified as appropriate weight, overweight or under-weight/having growth-faltering, were purposively selected and invited to participate in InDepth Interviews (IDIs). In total, 21 IDIs were conducted among the caregivers. The interviews were scheduled depending on the caregiver's convenience and were conducted in English by the principal investigator. Each interview lasted approximately 30 minutes and was audio-recorded. In addition, field notes were taken. The sample size was guided by the principle of saturation, that is, the point at which collection of new data does not yield any new information. Data were collected from September to December 2018.

\subsection{Data Analysis}

\subsection{Quantitative data}

Data were analyzed using the Statistical Package for Social Sciences (SPSS)version 21.0.

Proportions were estimated for categorical outcomes describing the knowledge and practice of caregivers as measured against WHO guidelines. Caregivers who scored 0-10 were considered as having poor knowledge on complementary feeding. Those who scored 11-15 were considered to have average knowledge while caregivers who scored 16-20 demonstrated good knowledge on complementary feeding practices. In the analysis of the complementary feeding practices, all the aforestated core indicators had to be met for a caregiver to be considered compliant with the WHO guidelines. The variable was then categorized in binary form (yes or no).

Factors affecting compliance with WHO guidelines such as socio-demographic characteristics were analyzed by descriptive statistics. Continuous data were summarized as mean or median scores. Chisquare test was used to investigate the relationship between predisposing, enabling and reinforcing factors and complementary feeding practices. Four variables were found to have a significant relationship with complementary feeding practices in the univariate analyses $(p<0.25)$. These were retained in the multivariate logistic regression model building process to predict compliance to complimentary feeding guidelines $(p<0.05)$.

\subsubsection{Qualitative data}


Data were audio-recorded and transcribed verbatim. Data were then analyzed using thematic framework analysis, as described by Ritchie and Spencer (10).

The principal investigator organized data from the original transcripts into charts with headings and subheadings derived from the constructed thematic framework. After charting the data according to the main themes, it was mapped and interpreted as a whole. The researcher reviewed the charts and research notes, comparing and contrasting the thoughts and experiences of different respondents. The mixed nature of this study i.e. quantitative and qualitative, allowed enhancement of the study's internal validity via triangulation. This was applied to predisposing factors such as: culture, religion and gender of the child which were assessed using both the questionnaire (quantitative) and the interview guide (qualitative). The consistency of evidence from the two sources, as opposed to reliance on a single source, was used to strengthen the interpretations made. Triangulation also helped to reduce researcher bias since findings were substantiated by data from multiple sources. Two members of the research team (CN and CK) independently coded the transcripts and generated themes. Any disagreement on codes and emerging themes was resolved through discussion. This process served to maximize the rigor and validity of the analysis.

\subsection{Ethical Considerations}

The AKUH-N Institutional Ethics Research Committee approved this study. An explanation of the purpose of the study was given to participants and interviews were conducted following written informed consent. Personal information was encrypted in order to maintain confidentiality.

\section{Results}

\subsection{Characteristics of the Study Population}

A total of 343 caregivers were approached at the well-baby clinic to participate in the study. Twenty three(6.7\%) did not meet inclusion criteria and 30 (8.8\%) declined to participate. The remaining 290 $(84.5 \%)$ were interviewed. The demographic characteristics of the caregivers and their infants are shown in Table 3.1 
Table 3.1

Characteristics of infants, young children and their caregivers

\begin{tabular}{|c|c|}
\hline Variable & Frequency (\%) \\
\hline \multicolumn{2}{|l|}{ Sex } \\
\hline Male & 147 (50.7) \\
\hline Female & 143 (49.3) \\
\hline \multicolumn{2}{|l|}{ Age } \\
\hline $9-12$ months & $188(64.8)$ \\
\hline $13-24$ months & $102(35.2)$ \\
\hline \multicolumn{2}{|l|}{ Sex } \\
\hline Female & $286(98.6)$ \\
\hline Male & $4(1.4)$ \\
\hline \multicolumn{2}{|l|}{ Religion } \\
\hline Catholic & 85 (29.3) \\
\hline Protestant & 179 (61.7) \\
\hline Islam & $16(5.5)$ \\
\hline None & $1(0.3)$ \\
\hline Other & $9(3.1)$ \\
\hline \multicolumn{2}{|l|}{ Education Level } \\
\hline None & $2(7)$ \\
\hline Primary & $2(7)$ \\
\hline Secondary & $12(4.1)$ \\
\hline College & $48(16.6)$ \\
\hline University & 226 (77.9) \\
\hline \multicolumn{2}{|l|}{ Occupation } \\
\hline Housewife & $22(7.6)$ \\
\hline Business & $66(22.8)$ \\
\hline Professional & 194 (66.9) \\
\hline Student & $5(1.7)$ \\
\hline
\end{tabular}




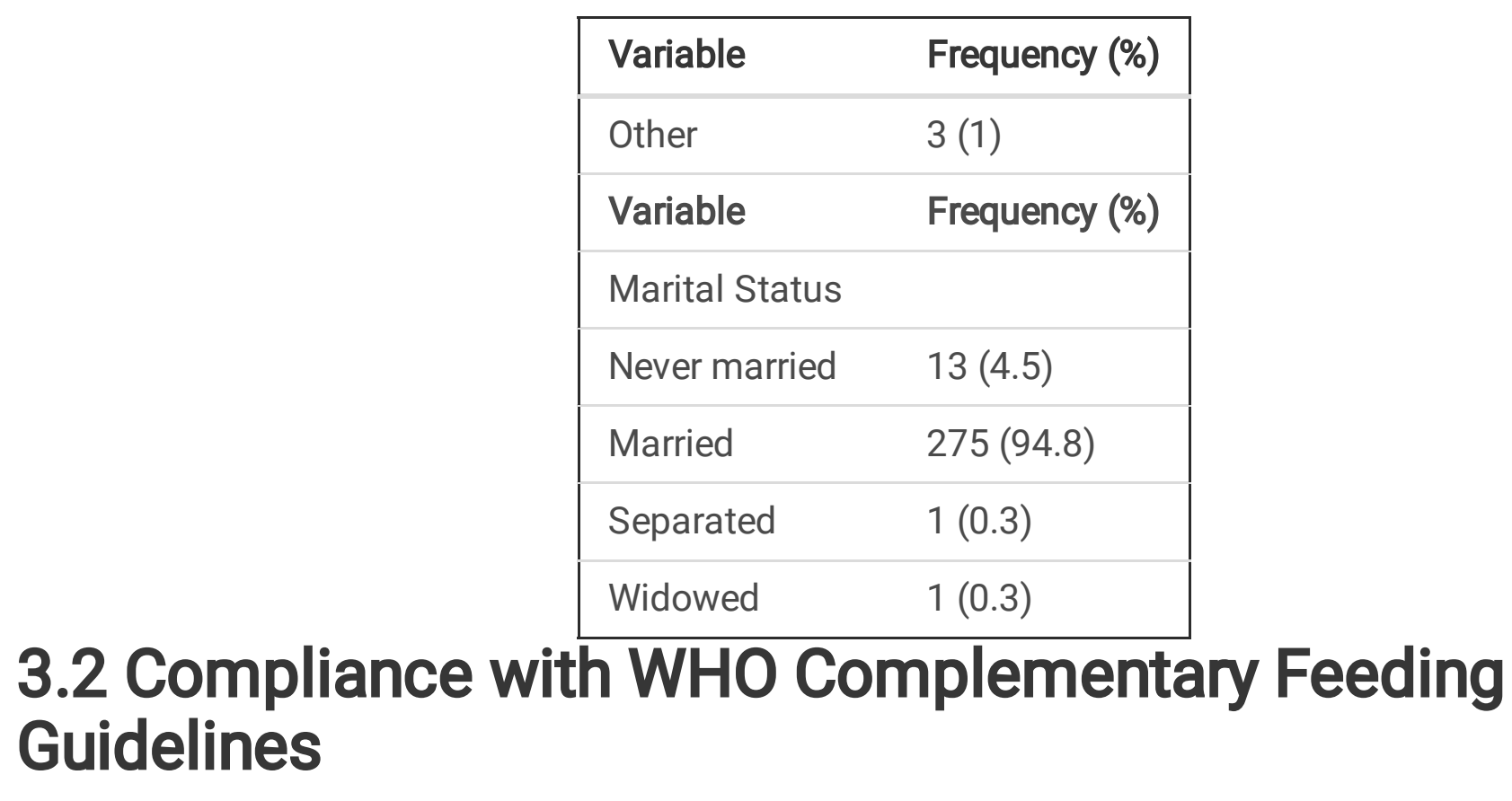

The rates of compliance with different aspects of the WHO complementary feeding guidelines are as tabulated in Table 3.2: 
Table 3.2

Compliance with WHO Complementary Feeding Guidelines

\begin{tabular}{|c|c|}
\hline Variable & Frequency (\%) \\
\hline \multicolumn{2}{|l|}{ Introduction of CF } \\
\hline Before 6 months & $40(13.8)$ \\
\hline At 6 months & $223(76.9)$ \\
\hline After 6 months & $27(9.3)$ \\
\hline \multicolumn{2}{|c|}{ Iron Rich food/supplement } \\
\hline Yes & $235(81)$ \\
\hline No & $55(19)$ \\
\hline \multicolumn{2}{|c|}{ Continued BF 6-24 months } \\
\hline Yes & $215(74.1)$ \\
\hline No & $75(25.9)$ \\
\hline \multicolumn{2}{|l|}{ Reasons BF stopped } \\
\hline Not enough milk & $24(32.4)$ \\
\hline Went back to work & $3(4.1)$ \\
\hline Child uninterested & $33(44.6)$ \\
\hline Never Breastfed & $1(1.4)$ \\
\hline Other & $13(17.6)$ \\
\hline \multicolumn{2}{|c|}{ Minimum Meal Diversity } \\
\hline Met & $266(91.7)$ \\
\hline Not Met & $24(8.3)$ \\
\hline \multicolumn{2}{|c|}{ Minimum Meal Frequency } \\
\hline Met & $276(95.2)$ \\
\hline Not Met & $14(4.8)$ \\
\hline \multicolumn{2}{|c|}{ Compliant with WHO Guidelines } \\
\hline Compliant & $125(43.1)$ \\
\hline Non Compliant & $165(56.9)$ \\
\hline
\end{tabular}

3.3 Nutritional Status of the Infants and Young Children 
Twenty (6.9\%) of the infants and young children were underweight, five (1.7\%) stunted and $25(8.6 \%)$ overweight. A comparison with the national average rates is displayed in Fig. 3.1

\subsection{Factors Affecting Compliance with WHO Complementary Feeding Guidelines}

Eight variables were studied as potential predictors of compliance. Of these, four had univariate $p$-values $<0.25$. These were the child's age and sex as well as the caregiver's age and education level. In multiple logistic regression analysis, the child's age was associated with compliance, and caregivers of children aged 9-12 months were more likely to be compliant relative to those with older children $(p<0.001)$. Caregivers with lower level of education (below college level) had a significantly reduced odds of compliance $(P=0.04)$ (Table 3.3). 
Table 3.3

Factors associated with compliance with WHO Complementary Feeding Guidelines

\begin{tabular}{|c|c|c|c|c|}
\hline \multirow[t]{2}{*}{ VARIABLE } & \multicolumn{2}{|c|}{ UNIVARIATE ANALYSIS } & \multicolumn{2}{|c|}{ MULTIVARIATE ANALYSIS } \\
\hline & Crude OR (95\% Cl) & p-value & Adjusted OR (95\% Cl) & p-value \\
\hline Child's Age & 1 & $<0.001$ & 1 & $<0.001$ \\
\hline 13-24 months & $3.3(1.9-5.5)$ & & $3.4(2.0-5.9)$ & \\
\hline \multicolumn{5}{|l|}{$9-12$ months } \\
\hline Caregiver's Age & 1 & 0.22 & 1 & 0.22 \\
\hline 30 and Above & $1.4(0.8-2.2)$ & & $1.4(0.8-2.5)$ & \\
\hline \multicolumn{5}{|l|}{ Below 30} \\
\hline No. of children & 1 & 0.93 & $\mathrm{~N} / \mathrm{A}$ & N/A \\
\hline More than 1 Child & $1.0(0.6-1.6)$ & & & \\
\hline \multicolumn{5}{|l|}{1 Child } \\
\hline Child's Sex & 1 & 0.07 & 1 & 0.07 \\
\hline Male & $0.7(0.4-1.0)$ & & $0.7(0.4-1.1)$ & \\
\hline \multicolumn{5}{|l|}{ Female } \\
\hline Attitude towards EBF & 1 & 0.34 & $\mathrm{~N} / \mathrm{A}$ & $\mathrm{N} / \mathrm{A}$ \\
\hline Positive Attitude & $0.8(0.4-1.4)$ & & & \\
\hline \multicolumn{5}{|l|}{ Negative Attitude } \\
\hline Caregiver's Occupation & 1 & 0.73 & $\mathrm{~N} / \mathrm{A}$ & $\mathrm{N} / \mathrm{A}$ \\
\hline Professional & $0.9(0.6-1.5)$ & & & \\
\hline \multicolumn{5}{|l|}{ Others } \\
\hline Caregiver's Education Level & 1 & 0.06 & 1 & 0.04 \\
\hline College Level \& above & $0.3(0.1-1.0)$ & & $0.2(0.05-0.8)$ & \\
\hline \multicolumn{5}{|l|}{ Below College Level } \\
\hline Caregiver's Knowledge Score & 1 & 0.51 & $\mathrm{~N} / \mathrm{A}$ & $\mathrm{N} / \mathrm{A}$ \\
\hline High & $0.9(0.5-1.4)$ & & & \\
\hline Average & & & & \\
\hline
\end{tabular}

The findings from the qualitative arm were analysed and grouped into six different themes as shown in Table 3.4 below. The respondents $(R)$ were given identifiers based on the order in which they were 
interviewed and the nutritional status of the child, that is, underweight (UW), average weight (AW) or overweight (OW).

Table 3.4

Factors affecting compliance to WHO Complementary Feeding Guidelines

\section{Theme Supportive Quotes}

Religious and Cultural Beliefs
'Where I come from, kids don't even eat fish. They will not eat...you know...chicken. And I've even seen it in homes. Kids are always on grains. They can either have beans/if at all, they are having any proteins, they'll have beans, kamande (lentils) or ndengu (green grams). You know, that kind of stuff. But I think I've tended to walk away from that.'(R10, UW)

Knowledge of Complementary Feeding Guidelines

'there was nothing specific. Yeah, it was more of ... The different foods, I mean, the different food types. When to start-you know the practical bit of give this. So I know I need to give food that is rich in vitamin, but which one? I need to give food that is rich in protein but which one? Yeah.' $(R 18,0 W)$

Access to information or guidelines

'I read two studies by John Hopkins that one of the indicators is not really 24 weeks or six months...if they have doubled their birth weight, before even the 24 weeks. It's still safe enough to start the solids.' (R02, OW)

'Online, has been very helpful. There is this app called BabyCenter. It has almost all the information you need to get about feeding your baby...' $(R 11, \mathrm{OW})$

Resource constraints

'If I wasn't working I'd have introduced him to very many types of food...because I'd have time. You know now, what the maid is used to cooking is what I tell her, "Cook that." So she cooks that.' (R13, AW)

We don't have beyond our seasonal vegetables and fruits, it's very difficult to get these others. They are very expensive... (R6, AW)

Support from health care workers and family

$$
\begin{aligned}
& \text { 'She [paediatrician] is very patient...I feel she's very particular on my child. Even } \\
& \text { without the hospital visit she has given me a leeway of just calling. Especially } \\
& \text { when we run out of the supplements of the milk, I am at liberty to call her at any } \\
& \text { time. And I can come to the hospital and pick milk at any time.'(R09, UW) } \\
& \text { 'I want to be that type of a dad who doesn't want to leave everything on the } \\
& \text { mother's side. So, if I'm allowed to step in and uhm...help in any way, even if its } \\
& \text { feeding, I don't hesitate.'(R01, AW) }
\end{aligned}
$$

Fear of allergies
'Yeah, I did have the fear of allergies, but what I did is, I sort of spaced them out probably a bit too much... and also focused more on plant proteins. I think with those I started immediately.' (R18, UW)

Respondents knew of cultural and religious restrictions, but did not apply them to their complementary feeding practices. In terms of knowledge of complementary feeding guidelines, caregivers seemed to have broad concepts on the feeding guidelines, but lacked clarity in specific and practical aspects. Concerning access to guidelines, respondents indicated that multiple sources of information were available to most care givers, including family members, the paediatrician and the internet. Another emergent theme was lack of resources, both money and time, which limited the kind of feeds offered, participation in preparing the meals, and clinic visits. Support from health care workers and family members was also noted to be an important contributor towards compliance to guidelines. Finally, fear of 
allergies emerged as a deterrent factor. Certain foods, notably animal proteins, were either delayed or avoided by caregivers due to fear of allergic reactions. This theme was frequent among caregivers with underweight infants.

\section{Discussion}

The overall level of compliance with the WHO guidelines for complementary feeding was higher than that reported nationally (5). This was possibly due to the fact that the study was based in a tertiary level hospital in a middle-class urban area. The KDHS 2014 (5) indeed reported that children in rural areas were less likely to be fed appropriately than their urban counterparts. Regionally, caregivers in Nairobi had the highest rate of adherence to Infant and Young Child Feeding (IYCF) practices. An increase in mother's education and increased household wealth were associated with more appropriate complementary feeding practices (5).

Age of the infant as well as caregiver's education level were found to be significantly associated with compliance with WHO guidelines on multivariate logistic regression. This is similar to previous studies that have reported that education level of the caregiver is associated with both complementary feeding practices and nutritional outcomes. For instance, lower maternal education and lower household wealth index were found to be the most consistent factors influencing inappropriate CF practices in the South Asian region (11). In Kenya, significant differences between malnourished children and children with normal nutritional status in relation to the education levels of their mothers and their fathers have been reported (6). In that study, the child was more likely to be malnourished if he/she came from a family where both parents had low and no education .

Gender of the child, as it affects feeding practices, was also explored in the qualitative arm of this study. Caregivers narrated the myth that 'boys feed more than girls', although this did not influence their feeding choices. This is in contrast to other studies which have reported gender bias in feeding. For instance, Kimani-Murage et al (7) found that male gender of the child was associated with earlier introduction of complementary feeds. The reason for this, though given anecdotally, was that boys did not seem to achieve satiety with breast milk alone. This fits into the forementioned myth that boys have higher nutritional demands than girls.

Cultural beliefs concerning complementary feeding practices were varied as caregivers came from different cultural backgrounds. Prohibitions, though freely quoted by caregivers, were mostly ignored because respondents felt that they were outdated. This is in contrast to a study in South Africa which found strict adherence to cultural beliefs (12). This is likely because the South African study was conducted in a rural setting, as opposed to this study which was done in an urban setting. However, some caregivers conceded that their practice had been affected by culture, albeit subconsciously. For example, there was a tendency to overfeed with certain starches such as potatoes and pumpkins and a hesitance to introduce some animal proteins like eggs. A commonly held cultural belief was that eggs, if given early, would cause a delay in the child's speech. However, on correlation with the quantitative data, it was found 
that almost half of the caregivers had given eggs to their children in the twenty-four hours prior to the study. Many caregivers also reported that their culture advocated for early introduction of complementary feeds. This, again, was not reflected in actual practice as most caregivers reported introducing complementary feeds at six months.

Caregivers in this study had access to multiple sources of information regarding complimentary feeding. Majority of them still considered the paediatrician their primary source of information and listed previous experience, the internet, relatives and friends as other influencers. The internet, in particular, served to provide supplementary information as well as support since it allowed for connection with other caregivers. Most caregivers attended the six-month clinic and received some nutritional advice. However, the qualitative aspect of this study revealed that there was inconsistency in the information given. This is likely due to lack of standardised protocol for complementary feeding at the well-baby clinic. The time constraint of a busy clinic also posed a challenge to the successful delivery of nutritional advice. This calls for a multidisciplinary approach to the problem, for example, involving nurses and nutritionists. Caregivers also lacked in-depth understanding about the importance of various guidelines. All these factors led to a disparity between the level of knowledge and full compliance with the guidelines. These findings are similar to those of a study done at Mbagathi District Hospital, Nairobi, that found lack of consistency in information delivered to caregivers by health care workers which led to a disparity between knowledge and practice (Otaigo, 2014).

The internet as a source of information was frequently mentioned. A variety of sources ranging from scientific research papers to online interactive sites to popular social media platforms like Facebook were quoted. The internet was seen as an authority by some caregivers but others approached it with more caution and confirmed the information therein with their healthcare provider. Plantinet al (14) in their review found that majority of parents went online in search of information or support. An important reason for this was weakened social support from caregiver's relatives and friends. Professionals had responded to this interest by offering online information as well as support. Benefits such as increased access to resources, without an added cost implication, and the ability to meet the demand for information round the clock were cited.

We found that caregivers were least compliant to the guidelines on continued breastfeeding to the age of two years. An increase in the age of the infant in this study was associated with discontinuation of breastfeeding $(P<0.001)$. This is similar to a study done in Tanzania $(15)$ where breast feeding rates were also reported to decrease with age. The two main reasons given for discontinuation were the child losing interest in breastfeeding and insufficient milk. This may be an indication of the need to continue lactation support beyond the immediate post-partum period and educate mothers on the importance of continued breastfeeding. Findings from the qualitative arm of this study underscored the role that lactation nurses played in encouraging mothers to continue breastfeeding. They followed mothers through phone calls and offered a holistic approach to lactation management, including addressing the psycho-social aspects. 
Majority of the caregivers in this study were professionals who cited time as a major challenge for clinic attendance, which meant less contact time with the healthcare workers. Time constraints also affected the extent to which some caregivers were able to plan, prepare and feed their children. There was, however, no significant association between profession and compliance with the WHO feeding guidelines. This may be due to the fact that the study was done in a tertiary hospital based in an urban setting and most caregivers were professionals. Peltoet a/ reviewed case studies on improving feeding practices and found that the issue of time as one of the most recurring themes. Caregivers often had competing demands on their time that created constraints on care-giving. This was true for both urban and rural settings, which was thought to be due to the fact that most caregivers today must also engage in an income-generating activity. The time factor was found to affect the selection and preparation of foods, as well as feeding styles (16). The structure of the well-baby clinic and the protocol developed must bear these factors in mind, if it is to impact the feeding practices of caregivers in our setting.

Majority of respondents in this study afforded private healthcare, either as part of an insurance scheme or out-of-pocket. Resources, however, are finite and this meant that some items were out of reach. This was especially true for caregivers who had underweight or allergy-prone babies and needed special formulas. Some parents wanted to expose their children to a wider variety of foods but found the cost of items that are not locally produced prohibitive. Socioeconomic status was not studied in the quantitative aspect of this study but has been found to be significantly associated with adherence to guidelines and nutritional outcomes in previous studies (6).

Fear of allergies emerged as a factor affecting introduction of various foods by caregivers, particularly protein rich foods. In some cases, the fear was well founded because of past experiences while in others, the fear was founded on suspicion or a myth. In cases where certain foods were completely restricted, suitable alternatives were not always available. In other cases, the caregiver opted to delay introduction of certain foods that are commonly known to be allergenic. There is currently no guideline on when potentially allergenic foods should be introduced. However, a recent randomized trial found that peanut and egg allergy were less prevalent in an early introduction group of three months than in the standard introduction group of six months (17). While the recommendation is certainly not to introduce allergenic foods in the third month of life, the findings suggest thatlate introduction of allergenic foods may actually predispose young children to development of allergies. This issue therefore needs to be addressed during development of complementary feeding guidelines.

Caregivers who worked outside the home had to rely on others to assist in preparing meals and feeding of their children. Good support in these areas positively affected feeding practices, as has also been reported in previous studies. A Gambian study, for instance, found that the social support network of mothers affected adherence to the infant care guidelines. The husband was reported as the most important of these support systems (18).

Regarding health-worker support, caregivers emphasized the important role played by nurses in giving holistic support, especially during the initial lactation period. When it came to complementary feeding, 
however, nurses were the least reported as an information source. This may be due to the fact that there were only two dedicated lactation nurses who focused mostly on establishing and supporting the initial breastfeeding period. The same emphasis is, however, not made for complementary feeding or continued breastfeeding. Numerous studies have shown the important role that can be played by health workers in influencing caregivers' feeding practices. A randomized trial on the efficacy of breastfeeding support provided by trained clinicians, for instance, showed that there was an improvement in breastfeeding outcomes with the intervention of a routine, preventive, outpatient visit (19). More training and support of all cadres of healthcare workers is needed to achieve this.

Lastly, it is of note that the proportion (8.6\%) of the infants and young children in this study who were overweight was double that reported nationally (4\%) by the KDHS 2014 (5). Childhood obesity is a growing concern, especially in low and middle income countries, with 38 million children under five reported to be overweight worldwide(20). Inappropriate complementary feeding has been linked to childhood obesity(21), which increases the long- term risk for adult obesity and associated cardiovascular diseases. More studies are needed in this area to establish the role of appropriate complementary feeding in preventing obesity.

\section{Conclusion}

The overall compliance with WHO complementary feeding guidelines was $43.1 \%$. Nutritional status outcomes rates were at $6.9 \%$ and for underweight, $1.7 \%$ and $8.6 \%$ for stunting and overweight respectively.

Factors positively associated with compliance with complementary feeding guidelines were younger age of the child, access to multiple sources of information as well as support from health workers and family. A below-college education level of the caregiver, lack of specific and practical guidelines, resource constraints and fear of allergies were the factors found to negatively impact compliance.

Health workers play an important role in influencing caregivers' feeding practices. As such, there is need to train and support nurses, nutritionists and all clinicians who work with children, to take up this role.

The areas identified for further research include: modifiable factors associated with childhood obesity, interventional studies of measures that would improve compliance to complementary feeding guidelines as well specifically studying borderline pre-terms who are known to have feeding difficulties.

\section{Declarations}

\section{Ethical Approval and Consent to participate}

Ethical approval to conduct this study was obtained from AKUH-N Ethical Review Committee Ref 2017/REC-88(v2). 
Consent was sought from the study subjects for this study and the hard copy forms retained by the Department of Paediatrics and Child Health.

\section{Consent for publication}

All the authors have read the manuscript before submission and give consent for publication

\section{Availability of data and materials}

Questionnaires, audio recordings, transcripts and notes were stored in a locked cabinet (hard copies) and in password protected computers. At the end of the study, all data was handed over to AKU Faculty of Health Sciences as per section 4.1.6(f) of the Faculty manual of research policies and procedures. The dataset(s) supporting the conclusions of this article is(are) included within the article (and its additional file(s).

\section{Competing Interests}

There are no conflicts of interest to declare.

\section{Funding}

Research Grant given by the Aga Khan University Hospital- Ref 2017/REC-88(v2).

\section{Author Contributions}

Catherine Njeru, Caroline Kathomi, Mary Slessor Limbe and Anthony Ngugi were all involved in the design of the study, analysis of results and the review of this manuscript. Catherine Njeru performed the study and wrote the manuscript

\section{Acknowledgements}

We acknowledge Dr. Wahu Gitaka for her invaluable insights and support as well as James Orwa who gave much needed support in the data analysis

\section{References}

1. UNICEF. Nutrition in the First 1, 000 Days. The State of the World's Children. 2012.

2. UNICEF. FROM THE FIRST HOUR OF LIFE Making the case for improved infant and young child feeding everywhere Key Findings 2016 Indicators Recommended Practices. 2016.

3. WHO. Indicators for assessing infant and young child feeding practices. Conclusions of a consensus meeting held 6-8 November 2007 in Washington, DC, USA. 2008.

4. WHO. Global Strategy on Infant and Young Child Feeding. World Health Organization. 2008.

5. Kenya National Bureau of Statistics. Kenya Demograpphic and Health Survey 2014. 2015. 
6. Bakalemwa R. Association Between Malnutrition and Feeding Practice Among children aged 6- 24 months at Mbagathi District Hospital, Kenya. The University of Nairobi; 2014.

7. Kimani-murage EW, Madise NJ, Fotso J, Kyobutungi C, Mutua MK, Gitau TM, et al. Patterns and determinants of breastfeeding and complementary feeding practices in urban informal settlements, Nairobi Kenya. BMC Public Health. 2011;11(1):396.

8. WHO. Complementary feeding. 2001.

9. Green LW, Kreuter MW. Health Program Planning An Educational and Ecological Approach. Fourth edi. New York: McGraw-Hill; 2005.

10. Richie J, Spencer L. Qualitative data analysis for applied policy research In: Bryman A, Burgess R (eds) Analysing Qualitative Data. Bryman A, Burgess RG, editors. London: Routledge; 1994.

11. Senarath, U., Agho, K. E., Akram, D., Godakange, S. S., Hazir, T., Jayawickrama, H. J, N., Kabir, I., Khanam, M., Patel A and O. Comparisons of complementary South, feeding indicators and associated factors in children aged 6-23 months across five Countries. Asian Matern child Nutr. 2012;8(s1):89-106.

12. Kruger R, Gericke GJ. A qualitative exploration of rural feeding and weaning practices , knowledge and attitudes on nutrition. Public Health Nutr. 2003;6(2):217-23.

13. Otaigo ML. Correlates of Complementary Feeding Practice Among Caregivers of Infants and Young Children Aged 6-24 Months At. The University of Nairobi; 2014.

14. Plantin L, Daneback K. Parenthood, information and support on the internet. A literature review of research on parents and professionals online. 2009;12:1-12.

15. Vitta BS, Benjamin M, Pries AM, Champeny M, Zehner E, Huffman SL. Original Article Infant and young child feeding practices among children under 2years of age and maternal exposure to infant and young child feeding messages and promotions in Dar es Salaam , Tanzania. 2016;12:77-90.

16. Pelto GH, Levitt E, Thairu L. Improving feeding practices: Current patterns, common constraints, and the design of interventions. 2003;24(1):45-82.

17. Perkin MR, Ph D, Logan K, Ph D, Tseng A, Raji B, et al. Randomized Trial of Introduction of Allergenic Foods in Breast-Fed Infants. 2016;1733-43.

18. Nabwera HM, Moore SE, Mwangome MK, Molyneux SC, Darboe MK, Camara-trawally N, et al. The influence of maternal psychosocial circumstances and physical environment on the risk of severe wasting in rural Gambian infants: a mixed methods approach. 2018;1-19.

19. Prospective PVA, Berchotteau M, Bouchon N, Schelstraete C, Vittoz J, Participants A. Efficacy of Breastfeeding Support Provided by Trained Clinicians During an Early, Routine, Preventive Visit: A Prospective, Randomized, Open Trial of 226 Mother-Infant Pairs. 2005;115(2).

20. UNICEF / WHO / World Bank Group. Joint Child Malnutrition Estimates Key findings. 2020;

21. Mameli C, Mazzantini S, Zuccotti GV. Nutrition in the First 1000 Days: The Origin of Childhood Obesity. 2016; 
Figures

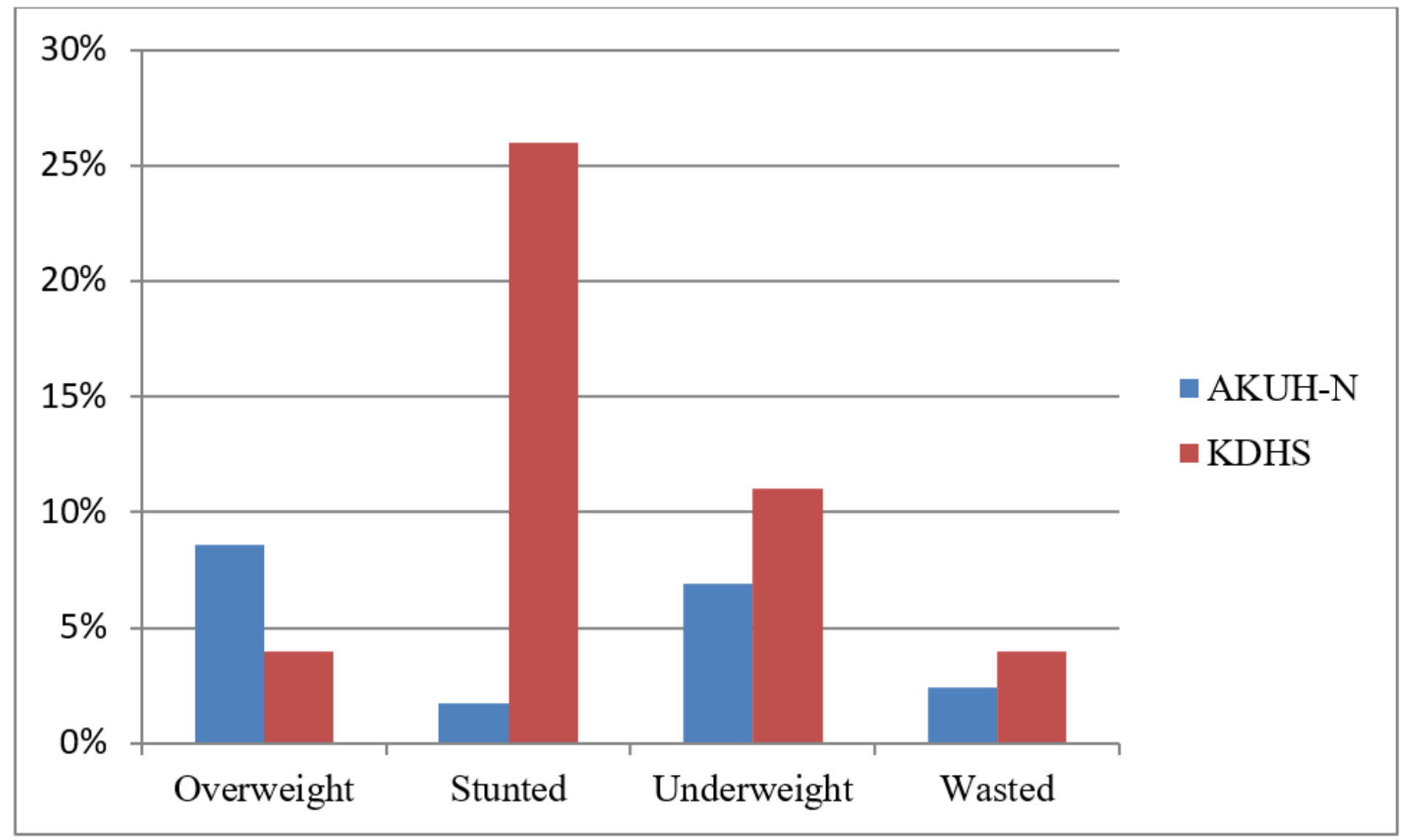

Figure 1

Comparison between AKUH-N and KDHS 2014 nutritional outcomes 